\section{Un mayor nivel de actividad física se asocia a una menor prevalencia de factores de riesgo cardiovascular en Chile: resultados de la Encuesta Nacional de Salud 2009-2010}

\author{
CARLOS CELIS-MORALES ${ }^{1,2, a}$, CARLOS SALAS ${ }^{3, \mathrm{~b}}$, \\ CRISTIAN ÁLVAREZ ${ }^{4, c}$, NICOLÁS AGUILAR FARÍAS ${ }^{5, \mathrm{~d}}$, \\ RODRIGO RAMÍREZ CAMPILLOS ${ }^{6, \mathrm{e}}$, JAIME LEPPE ${ }^{7, \mathrm{f}}$, \\ CARLOS CRISTI-MONTERO ${ }^{8,9, \mathrm{~g}}$, XIMENA DÍAZ MARTÍNEZ $^{10, \mathrm{~h}}$, \\ ELIANA DURAN ${ }^{11, i}$, ANA MARÍA LABRAÑ ${ }^{11, i}$, \\ MARÍA ADELA MARTÍNEZ ${ }^{12, j}$, ANA MARÍA LEIVA ${ }^{13, \mathrm{k},}$ \\ NAOMI WILLIS ${ }^{2,1}$
}

\section{Higher physical activity levels are associated with lower prevalence of cardiovascular risk factors in Chile}

Background: Little is known about the relationship between physical activity (PA) and cardiovascular risk factors in the Chilean population. Aim: To investigate the association between different levels and intensities of $P A$ and the prevalence of cardiovascular $(\mathrm{CV})$ risk factors in Chilean adults. Material and Methods: Data from the National Health Survey 2009-10 including 5157 participants, provided by the Epidemiology Department of the Ministry of Health, was analyzed in this study. The prevalence of type 2 diabetes mellitus, hypertension, metabolic syndrome and dyslipidemia were determined using international criteria. PA levels were determined using the Global Physical Activity Questionnaire (GPAQ v2) and different levels of $P A$ were derived from it (transport-related, moderate and vigorous $P A)$. Quartiles of $P A$ were determined to investigate the association between $P A$ and cardiovascular risk factors. Results: Twenty three percent of women and $17.1 \%$ of men did not meet the PA recommendation ( $\geq 600$ METs.min.week-1). When prevalence of $C V$ risk factors were compared between inactive individuals ( $<600$ METs.min.week-1) and active individuals $(\geq 9500$ METs.min.week-1) a significantly lower prevalence of diabetes mellitus (6.2\% and 10\%), hypertension (18.0\% and $12.4 \%$ ) and metabolic syndrome (8.9\% and $12.1 \%)$ for women and men, respectively, was found in the active participants. Similar results were found for high versus low transport-related PA. Conclusions: Increasing levels of PA are associated with a significantly lower frequency of cardiovascular risk factors in Chilean adults.

(Rev Med Chile 2015; 143: 1435-1443)

Key words: Cardiovascular diseases; Motor; Risk factors.
${ }^{1}$ BHF Glasgow Cardiovascular Research Centre, Institute of Cardiovascular and Medical Science, Glasgow University, Glasgow, United Kingdom. ${ }^{2}$ Human Nutrition Research Centre, Institute of Cellular Medicine, Newcastle University, Newcastle upon Tyne, United Kingdom. ${ }^{3}$ Departamento de Educación Física. Facultad de Educación. Universidad de Concepción. Concepción, Chile.

${ }^{4}$ Centro de Salud Familiar Tomás Rojas de Los Lagos, Chile.

${ }^{5}$ Departamento de Educación Física, Deporte y Recreación, Universidad de Temuco, Temuco, Chile.

${ }^{6}$ Departamento de Educación Física, Universidad de Los Lagos, Osorno, Chile. ${ }^{7}$ Escuela de Kinesiología, Facultad de Medicina, Universidad del Desarrollo, Santiago, Chile.

${ }^{8}$ Grupo IRyS. Escuela de Educación Física. Pontificia Universidad Católica de Valparaíso, Valparaíso, Chile.

`Universidad Autónoma de Chile, Chile. ${ }^{10} \mathrm{Grupo}$ de Investigación Calidad de Vida, Universidad del Bio-Bío, Chillán, Chile. ${ }^{11}$ Departamento de Nutrición, Facultad de Farmacia, Universidad de Concepción, Concepción, Chile.

${ }^{12}$ Instituto de Farmacia, Facultad de Ciencias, Universidad Austral de Chile, Valdivia, Chile. ${ }^{13}$ Instituto de Anatomía, Histología y Patología, Facultad de Medicina, Universidad Austral de Chile, Valdivia, Chile.

aprofesor de Educación Física, Doctor en Ciencias Cardiovasculares y Biomédicas. bProfesor de Educación Física, Magíster en Educación Física, Candidato a PhD en Educación.

'Profesor de Educación Física, Magíster en Educación Física.

${ }^{\mathrm{d} K i n e s i o ́ l o g o, ~ M a g i ́ s t e r ~ e n ~ C i e n c i a s . ~}$

eprofesor de Educación Física, Doctor en Ciencias de la Salud.

${ }^{\text {}}$ Kinesiólogo, Magíster en Epidemiología. gProfesor de Educación Física, Doctor en Ciencias de la Actividad Física y del Deporte.

hProfesora de Educación Física, Magíster en Educación.

'Nutricionista, Magíster en Nutrición. 'Bioquímico, Magíster en Nutrición y Dietética.

kProfesor de Biología, Química y Ciencias, Magíster en Neurociencias y Salud Mental.

'Bióloga, PhD Ciencias Biológicas.

Recibido el 3 de junio de 2015, aceptado el 25 de septiembre de 2015 .

Correspondencia a:

Dr. Carlos Celis-Morales

BHF Glasgow Cardiovascular Research Centre

126 University Avenue

Glasgow University

Glasgow

United Kingdom

G12 8TA

Teléfono: + (0)4401413304201

carlos.celis@glasgow.ac.uk 
M undialmente, la falta de practica de actividad física (AF) se ha convertido en uno de los factores de riesgo más importante en el desarrollo de enfermedades crónicas no transmisibles (ECNTs) $)^{1,2}$. Ser inactivo es causante de $6 \%$ a $10 \%$ de enfermedad coronaria, hipertensión arterial, diabetes mellitus tipo 2, y cáncer de mama y de colon ${ }^{1}$.

Más aun, la inactividad física se asocia a $9 \%$ de muertes prematuras en el mundo, equivalente a 5,3 millones de muerte por año ${ }^{1}$. Se estima que al reducir la inactividad física entre $10 \%$ y $25 \%$ a nivel mundial, el número de muertes prematuras se reduciría entre 0,5 y 1,3 millones por año ${ }^{1}$. Esto posiciona a la inactividad física como un factor de riesgo similar al tabaquismo, causante de más de 5 millones de muertes a nivel mundial por año ${ }^{1,2}$.

Los beneficios de la práctica de $\mathrm{AF}$ no es algo nuevo; sociedades antiguas (China 2600 a.C. o Hipócrates 400 a.C. ya creían en la práctica de AF como una herramienta esencial para la salud ${ }^{1}$ ), pero, sólo a partir de 1953, se produce la primera evidencia científica que reporta los beneficios de la práctica de $\mathrm{AF}$ en la reducción de enfermedades coronarias $^{3}$. A pesar que existe suficiente evidencia científica que confirma los beneficios de la práctica de AF, actualmente $31,1 \%$ de la población adulta a nivel mundial no realiza AF suficiente, con prevalencias de inactividad que van desde $17 \%$ en sureste asiático, $43 \%$ en las Américas ${ }^{4}$ a 19,8\% en la población chilena ${ }^{5}$.

Si bien la práctica de AF se asocia a una reducción en el desarrollo de enfermedades cardiovasculares (ECVs), se desconoce la naturaleza de esta asociación en la población chilena. Conocer de qué forma se relaciona el incremento de diferentes intensidades de AF con la prevalencia de factores de riesgo cardiovascular (FRCV), podría ser de gran utilidad para entender qué tipo de actividades y cuanta AF debería realizarse para reducir las ECVs. Lo anterior podría traducirse en la creación de recomendaciones e implementación de políticas públicas orientadas a la práctica de AF. En consecuencia, el objetivo de este estudio fue investigar la asociación entre distintos niveles e intensidades de AF con la prevalencia de FRCV en población adulta en Chile.

\section{Materiales y Métodos}

La Encuesta Nacional de Salud 2009-2010 presenta resultados que permiten ser analizados de acuerdo a nuestros propósitos.

\section{Diseño de la encuesta}

La muestra seleccionada comprende todos los participantes de la Encuesta Nacional de Salud (ENS 2009-10) desarrollada entre octubre del año 2009 y septiembre de $2010^{5}$. La base de datos fue proporcionada por el Departamento de Epidemiología del Ministerio de Salud. La ENS 2009-10 corresponde a un estudio de prevalencia realizado en hogares en una muestra nacional, probabilística, estratificada y multietápica de 5.412 personas mayores de 15 años, con representatividad nacional, regional, y área urbano/rural. Para cada individuo se consideró un factor de expansión correspondiente al inverso de la probabilidad de selección del individuo, lo que permite corregir los resultados muestrales considerando la probabilidad desigual de selección de cada entrevistado, dado el diseño muestral y la post-estratificación demográfica según proyecciones censales a enero de 2010.

Los participantes firmaron un consentimiento informado y sus resultados les fueron devueltos con recomendaciones y derivación según correspondía ${ }^{5}$. El protocolo de estudio que definió la encuesta fue aprobado por el Comité de Ética de Investigación de la Escuela de Medicina de la Pontificia Universidad Católica de Chile.

\section{Mediciones antropométricas y metabólicas}

Las muestras de sangre de los participantes en ayuno fueron obtenidas por una enfermera entrenada, siguiendo protocolos estandarizados a nivel nacional ${ }^{5}$. Los marcadores metabólicos de glicemia, perfil lipídico (triglicéridos, colesterol HLD, colesterol LDL, colesterol total) y presión arterial fueron medidos con métodos estandarizados y previamente descritos en la ENS 2009-10 Los puntos de corte utilizados para la detección de enfermedades cardiovasculares fueron los siguientes: hipertensión arterial (PAS $\geq 140 \mathrm{mmHg}$ o $\mathrm{PAD} \geq 90 \mathrm{mmHg}$ ), diabetes mellitus (glicemia en ayuno $\geq 126 \mathrm{mg}^{-\mathrm{dl}^{-1}}$ ), colesterol total elevado $\left(>200 \mathrm{mg}^{-\mathrm{dl}^{-1}}\right)$, colesterol HDL disminuido $(<40$ mg.dl ${ }^{-1}$ en hombres $\mathrm{y}<50 \mathrm{mg} \cdot \mathrm{dl}^{-1}$ en mujeres), triglicéridos elevados $\left(>150 \mathrm{mg} \cdot \mathrm{dl}^{-1}\right)$. El síndrome metabólico fue determinado utilizando el criterio de la IDF-ATP ${ }^{5,6}$, que requiere al menos 3 de los siguientes 5 criterios para su clasificación: presión 
arterial $(>130 / 85 \mathrm{mmHg}$ ), circunferencia cintura elevada ( $88 \mathrm{~cm}$ hombres y $83 \mathrm{~cm}$ mujeres), colesterol HDL $(<40 \mathrm{mg} / \mathrm{dl}$ en hombres o $<50$ en mujeres), glicemia elevada $\left(>100 \mathrm{mg}^{-\mathrm{dl}^{-1}}\right)$, triglicéridos elevados $\left(>150 \mathrm{mg} \cdot \mathrm{dl}^{-1}\right)$.

\section{Clasificación de actividad física}

Los niveles de actividad física (AF) de la población fueron determinados con el cuestionario "Global Physical Activity Questionnaire" (GPAQ $\mathrm{v} 2)^{7}$, el cual ha sido validado internacionalmente ${ }^{8}$ y en población latina ${ }^{9}$. Los niveles de sedentarismo fueron determinados mediante el auto-reporte de tiempo destinado a actividades que involucren estar sentado o reclinado durante el tiempo libre o de trabajo (Ej. tiempo sentado frente al computador, viendo televisión, viajando en bus, tren o auto, etc.).

El tiempo destinado a las actividades físicas relacionadas al transporte (ej. caminar, andar en bicicleta) y actividades de intensidad moderada o vigorosa realizadas en el tiempo libre y en el trabajo, fueron determinadas según la guía de análisis de GPAQ ${ }^{7}$. Para determinar los niveles de actividad física total, las variables fueron expresadas en METs (metabolic-energy-equivalents), que es una unidad que se utiliza frecuentemente para estimar la intensidad de distintos tipos de actividad física. Se considera que 1 MET es equivalente al gasto energético basal de una persona en reposo (equivalente a $1 \mathrm{kcal} / \mathrm{kg} / \mathrm{h})^{10}$. Se consideró como punto de corte para inactividad física un gasto energético menor a $600 \mathrm{METs} / \mathrm{min} / \mathrm{semana}$, según las recomendación de la Organización Mundial de la Salud (OMS) y especificaciones en la guía de análisis de $\mathrm{GPAQ}^{7,11}$.

\section{Análisis estadístico}

Las tasas de prevalencia y medias se calcularon utilizando los factores de expansión conforme al diseño complejo de la encuesta y ajustando la muestra a la demografía chilena a junio de $2010^{5}$. Para diferencias de género en variables de tipo continua, se utilizó la prueba de t-test para muestras independientes. Para variables de tipo categóricas se utilizó el test de Chi-cuadrado $\left(\chi^{2}\right)$. Se aplicó el análisis de Generalized Linear Model para la interacción entre cuartiles de actividad física y sexo y su efecto en la prevalencia de FRCV. $\mathrm{El}$ análisis de tendencia (trend) fue determinado con regresiones logísticas. Para todos los análisis se utilizó el módulo de análisis de muestras complejas del programa STATA SE v14. El nivel de significancia fue definido como $\mathrm{p}<0,05$. Todos los resultados fueron estimados utilizando muestras expandidas según la ENS 2009-105.

\section{Resultados}

De la muestra total incluida en la ENS 2009-10 $(\mathrm{n}=5.416) 95 \%$ y $87 \%$ de la muestra tenía información en AF ( $\mathrm{n}=5.157)$ y marcadores metabólicos $(n=4.700)$ disponible, respectivamente. $\mathrm{La}$ edad promedio de la muestra fue 41,6 años; $59,5 \%$ eran mujeres (Tabla 1).

Los niveles de AF reportados eran significativamente mayores en hombres que en mujeres. El $23,1 \%$ y 17,1 de mujeres y hombres, respectivamente eran inactivos (realizaban menos de $600 \mathrm{METs} / \mathrm{min} / \mathrm{semana}$ ). Por otra parte, $70,1 \%$ y $34,8 \%$ de la población reportó no realizar ningún tipo de AF de intensidad vigorosa o de transporte (caminar o andar en bicicleta), respectivamente. Cuando se analizó el tiempo destinado a diferentes intensidades de AF (Figura 1), se encontró que del total de tiempo reportado, $48 \%$ y $54 \%$ de este tiempo en hombres y mujeres, respectivamente, se utilizaba en actividades de tipo sedentarias.

Las prevalencias de FRCV según sexo se presentan en la Tabla 2. Las prevalencias de hipertensión arterial, síndrome metabólico y triglicéridos elevados fueron significativamente mayores en hombres que en mujeres. No obstante, la prevalencia de colesterol HDL disminuido fue significativamente mayor en mujeres que en hombres. No se encontraron diferencias significativas entre sexos para diabetes mellitus tipo 2 o colesterol total elevado.

La asociación entre niveles de AF moderada o intensa y prevalencia de FRCV es reportada en la Figura 2. Se encontró una interacción significativa entre sexo ${ }^{\star} \mathrm{AF}$ para la prevalencia de colesterol HDL disminuido e hipertensión (Figura 2). El incremento de los niveles de AF reducía significativamente la prevalencia de colesterol HLD-disminuido en hombres pero no en mujeres. Si bien no se encontraron interacciones significativas entre sexo ${ }^{\star} \mathrm{AF}$ para diabetes mellitus, y síndrome metabólico, los resultados mostraron una tendencia significativa $(\mathrm{p}<0,002)$ a disminuir la prevalencia de diabetes mellitus e hipertensión al incrementar 

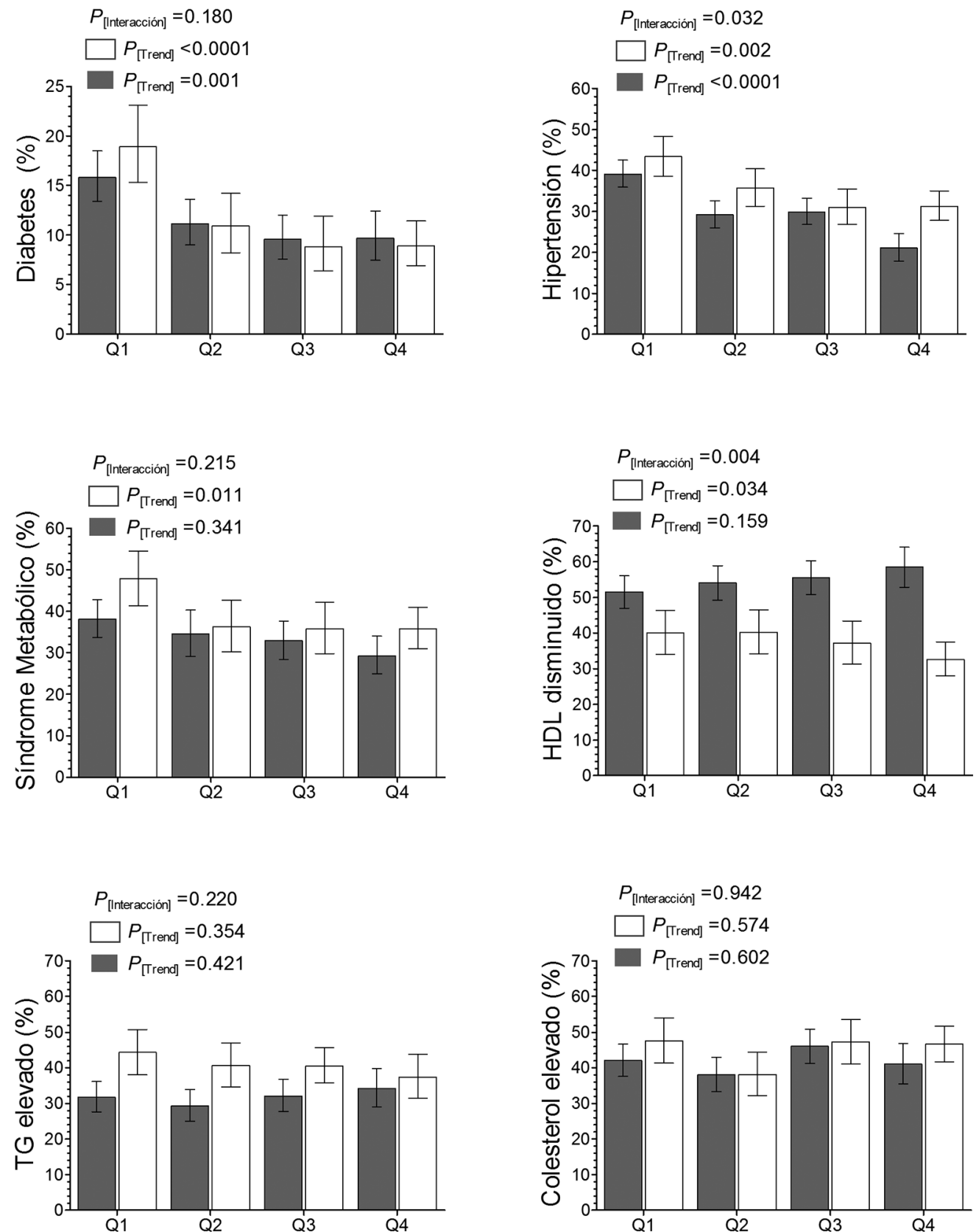

Cuartiles de Actividad Física Moderada/Vigorosa (METs.min.semana ${ }^{-1}$ )

Mujeres Hombres

Figura 1. Distribución del tiempo destinado a diferentes intensidades de actividad Física. 
Actividad física y factores de riesgo cardiovascular - C. Celis-Morales et al

Tabla 1. Características socio-demográficas, antropométricas y de actividad física según sexo

\begin{tabular}{|c|c|c|c|c|}
\hline Variables & Total & Mujeres & Hombres & $\begin{array}{c}\text { Valor p } \\
\text { (sexo) }\end{array}$ \\
\hline $\begin{array}{l}\text { Socio-demográficas } \\
\mathrm{n} \\
\text { Edad (años) }\end{array}$ & $\begin{array}{c}5.157 \\
41,6 \pm 0,4\end{array}$ & $\begin{array}{c}3.073 \\
42,5 \pm 0,5\end{array}$ & $\begin{array}{c}2.084 \\
40,7 \pm 0,6\end{array}$ & $<0,0001$ \\
\hline $\begin{array}{l}\text { Antropométricas } \\
\text { Peso }(\mathrm{kg}) \\
\text { Talla }(\mathrm{m}) \\
\text { IMC }\end{array}$ & $\begin{array}{c}72,2 \pm 0,3 \\
1,63 \pm 0,003 \\
27,4 \pm 0,2\end{array}$ & $\begin{array}{c}72,2 \pm 0,3 \\
1,56 \pm 0,003 \\
27,8 \pm 0,3\end{array}$ & $\begin{array}{c}77,4 \pm 0,5 \\
1,70 \pm 0,003 \\
26,9 \pm 0,2\end{array}$ & $\begin{array}{l}<0,0001 \\
<0,0001 \\
<0,0001\end{array}$ \\
\hline 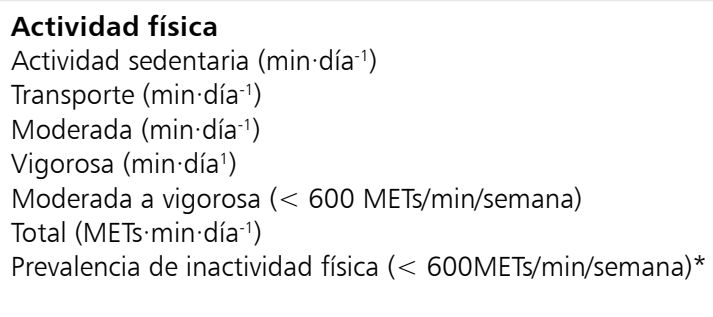 & $\begin{aligned} 211,2 & \pm 2,4 \\
48,9 & \pm 1,2 \\
103,7 & \pm 2,0 \\
50,1 & \pm 1,6 \\
816,3 & \pm 16,2 \\
1.014,1 & \pm 17,2 \\
20,2 & \\
(18,4 & \text { a } 22,2)\end{aligned}$ & $\begin{aligned} 202,9 & \pm 3,1 \\
45,6 & \pm 1,4 \\
96,6 & \pm 2,5 \\
31,1 & \pm 1,5 \\
635,4 & \pm 16,7 \\
841,2 & \pm 28,6 \\
23,1 & \\
(22,6 & \text { a } 25,9)\end{aligned}$ & $\begin{aligned} & 223,3 \pm 3,9 \\
& 53,7 \pm 1,9 \\
& 114,2 \pm 3,5 \\
& 78,2 \pm 3,3 \\
& 1083,2 \pm 30,8 \\
& 1.014,1 \pm 17,2 \\
& 17,1 \\
&(14,2 \text { a } 20,3)\end{aligned}$ & $\begin{aligned} & 0,0001 \\
& 0,002 \\
< & 0,0001 \\
< & 0,0001 \\
< & 0,0001 \\
< & 0,0001 \\
< & 0,0001\end{aligned}$ \\
\hline
\end{tabular}

Los datos son presentados como promedio \pm error estándar del promedio para variables continuas y como prevalencia (95\% IC) para variables categóricas*. Diferencias significativas entre sexo fueron estimadas mediante t-test para muestras no pareadas y $\chi^{2}$ test para variables categóricas.

Tabla 2. Prevalencia de factores de riesgo cardiovascular según sexo

\begin{tabular}{|lcccc|}
\hline Variables & \multicolumn{1}{c}{ Total } & Mujeres & Hombres & Valor p \\
\hline Diabetes mellitus & $9,4(8,1$ a 10,9$)$ & $10,4(8,7$ a 12,5$)$ & $8,4(6,6$ a 10,6$)$ & 0,771 \\
\hline Hipertensión & $26,9(24,9$ a 29,1$)$ & $25,3(22,8$ a 28,0$)$ & $28,7(25,5$ a 32,1$)$ & 0,002 \\
\hline Síndrome metabólico & $30,4(27,4$ a 33,6$)$ & $28,9(25,2$ a 32,8$)$ & $32,1(27,3$ a 37,3$)$ & 0,010 \\
Colesterol HDL disminuido & $45,4(42,1$ a 48,7$)$ & $52,8(48,7$ a 57,0$)$ & $37,6(32,7$ a 42,9$)$ & $<0,0001$ \\
\hline TG elevados & $31,2(28,2$ a 34,4) & $27,1(23,6$ a 30,8 & $35,6(30,7$ a 40,7$)$ & $<0,0001$ \\
Colesterol total elevado & $38,5(35,4$ a 41,8) & $38,1(34,2$ a 42,1) & $39,0(34,1$ a 44,2$)$ & 0,083 \\
\hline
\end{tabular}

Los datos son presentados como prevalencia $\left(95 \%\right.$ IC). Diferencias significativas entre sexo fueron analizadas mediante $\chi^{2}$ test. Prevalencias fueron estimadas con el factor de expansión sugerido por la ENS 2009-10.

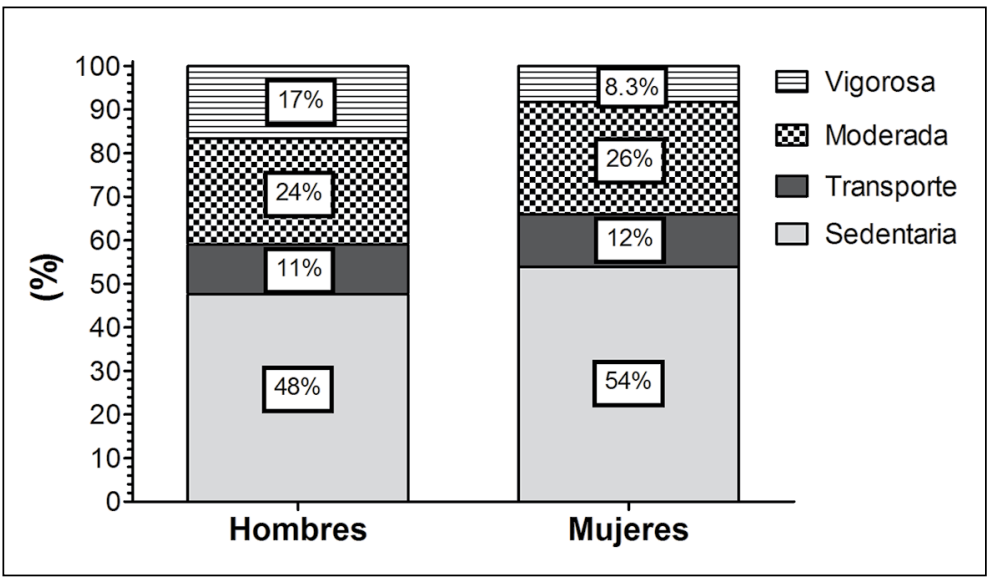

Figura 2. Prevalencia de factores de riesgo cardiovascular según nivel de actividad física de intensidad moderada/ vigorosa. Datos son presentados como prevalencia y sus respectivos intervalos de confianza $(95 \%$ IC). Los puntos de cortes para cuartiles de AF fueron expresados en METs.min.semana-1 según el tiempo reportado en $\mathrm{AF}$ de intensidades moderada a intensa (Q1: < 600 METs. min semana ${ }^{-1}$; Q2: 600 a 2.999 METs. min semana ${ }^{-1}$; Q3: 3000 a 9499 METs. min semana ${ }^{-1}$; Q4: $\geq 9.500$ METs.min. semana $\left.^{-1}\right)$. Valores $\mathrm{p}$ fueron calculados para la interacción entre sexo ${ }^{*}$ Cuartiles de AF, y para tendencia "Trend" según sexo. 

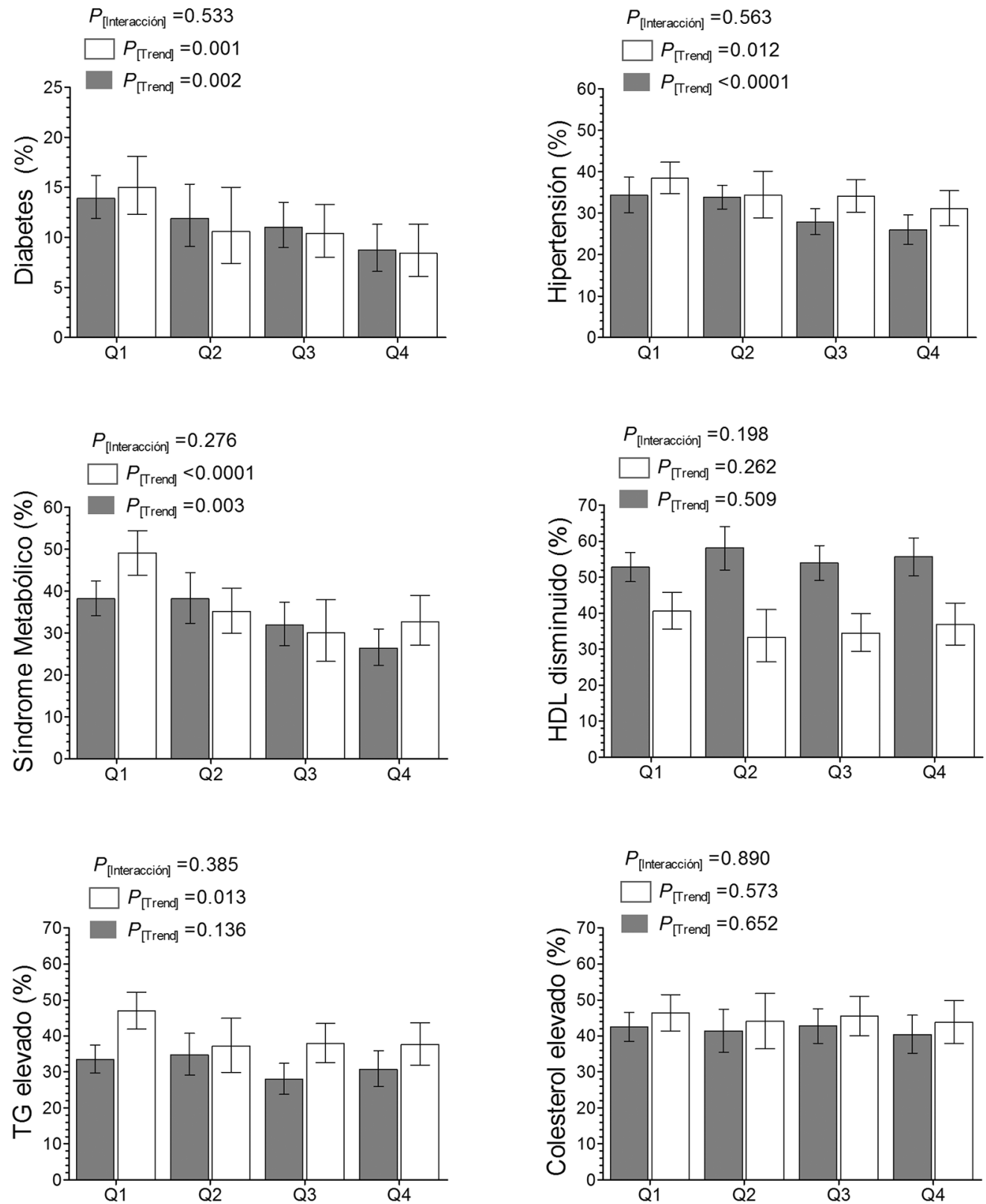

Cuartiles de Actividad Física de Transporte (min.semana ${ }^{-1}$ )

Hombres

Figura 3. Prevalencia de factores de riesgo cardiovascular según nivel de actividad física asociada a transporte. Datos son presentados como prevalencia y sus respectivos intervalos de confianza (95\% IC). Los puntos de cortes para cuartiles de AF de transporte (caminar y andar en bicicleta) fueron expresados en min.semana-1 según el tiempo reportado (Q1: < 10 min. semana $^{-1}$; Q2: 10 a 119 min.semana ${ }^{-1}$; Q3: 120 a 419 min.semana-1 ${ }^{-1}$ Q4: $\geq 420$ min.semana $\left.{ }^{-1}\right)$. Valores $p$ fueron calculados para la interacción entre sexo*Cuartiles de AF, y para tendencia "Trend" según sexo. 
los niveles de AF moderada/intensa en mujeres y hombres. La prevalencia de síndrome metabólico sólo disminuyó significativamente en hombres con el incremento de los niveles de AF (Figura 2). No se encontró ninguna asociación significativa entre AF y triglicéridos elevado o colesterol total elevado.

$\mathrm{Al}$ analizar la asociación entre AF de transporte (caminar o andar en bicicleta) con la prevalencia de FRCV, no se encontraron interacciones significativas entre sexo ${ }^{\star} \mathrm{AF}$ (Figura 3 ); sin embargo, la prevalencia de diabetes mellitus, hipertensión y síndrome metabólico se reducía significativamente al incrementar los niveles de AF de transporte, tanto en mujeres como en hombres. En cuanto a la prevalencia de triglicéridos elevados, ésta se redujo significativamente en hombres pero no en mujeres (Figura 3).

\section{Discusión}

\section{Principales resultados}

El principal resultado de este estudio fue la asociación inversa entre el incremento de AF y la reducción de la prevalencia de FRCV, tales como diabetes mellitus, hipertensión, síndrome metabólico y colesterol HDL disminuido. Estos resultados revelan que un incremento ligero en los niveles de AF de intensidad moderada/vigorosa, como lo es desplazarse del cuartil 1 "Niveles bajos de actividad física (<600 METs/min/semana)" al cuartil 2 (600-2999 METs/min/semana) induce una reducción importante en la prevalencia de diabetes mellitus $(-4,7 \%$ y $-8 \%)$, hipertensión arterial (HTA) (-9,9\% y $-7,7 \%)$, y síndrome metabólico $(-3,6 \%$ y $-11,7 \%)$, para mujeres y hombres, respectivamente. Para lograr estos beneficios, una persona tendría que caminar 25 min por día o remplazar la utilización del ascensor por subir las escaleras $10 \mathrm{~min}$ por día.

\section{Comparación con otros estudios}

Los hallazgos encontrados concuerdan con estudios previos donde se ha reportado una relación inversa entre AF y prevalencia de FRCV ${ }^{12-15}$. También estos resultados concuerdan con las recomendaciones internacionales de AF, que promueven la realización de al menos $600 \mathrm{METs} / \mathrm{min} / \mathrm{semana}$ de AF de intensidad moderada o intensa ${ }^{11}$. Si bien mayores niveles de AF se asocian a un menor riesgo cardiovascular ${ }^{1,13}$, los resultados muestran que con sólo cumplir con la recomendación de $\mathrm{AF}$, ya se observan importantes reducciones en la prevalencia de FRCV. Al comparar las prevalencias entre individuos con bajos niveles de AF (Cuartil 1) versus aquellos con altos niveles de AF (cuartil 4), se encontró una reducción de $6,2 \%$ y $10 \%$ en diabetes mellitus, $18 \%$ y $12,4 \%$ en HTA y $8,9 \%$ y $12,1 \%$ en síndrome metabólico, en mujeres y hombres, respectivamente. Lo cual demuestra que a mayor practica de AF mayor serán los beneficios a nivel cardiovascular.

A pesar que las recomendaciones internacionales de AF están basadas en actividades de intensidad moderada o vigorosa, estos resultados revelan que caminar o andar en bicicleta, también podrían ser un tipo de AF efectiva en la reducción de la prevalencia de FRCV. Al comparar las prevalencias entre individuos con bajos niveles de AF de transporte (Cuartil 1) versus altos niveles de AF (cuartil 4), se encontró una reducción de 5,2\% y $6,6 \%$ en diabetes, $8,4 \%$ y $7,4 \%$ en HTA y $11,8 \%$ y $16,4 \%$ en síndrome metabólico, en mujeres y hombres, respectivamente. Observaciones similares han sido reportadas en estudios internacionales que comparan los beneficios de actividades física ligera, como lo es caminar, con actividades físicas de intensidad moderada o vigorosa ${ }^{12,14}$, lo cual fortalece la evidencia respecto a que actividades cotidianas como caminar, si se asocian a beneficios cardiovasculares ${ }^{16}$. En consecuencia, este tipo de actividades deberían ser incorporadas en políticas públicas orientadas a la promoción de actividad física, ya que incrementar el tiempo que destinamos a caminar podría ser más fácil de ser incorporado en nuestros estilos de vida que actividades de intensidades moderadas o intensas ${ }^{17}$.

\section{Fortalezas y limitaciones del estudio}

Una de las fortalezas de este estudio es la representatividad nacional y escala de la ENS 2019-10. También se encuentra como fortaleza la medición de marcadores metabólicos para establecer la prevalencia de FRCV, la cual fue realizada en laboratorios certificados. No obstante, una de las limitantes de este estudio es la medición de AF a través de cuestionarios. Si bien GPAQ ha sido validado internacionalmente ${ }^{8,9}$, existe evidencia que la medición de AF a través de cuestionarios trae consigo una sobre estimación de los niveles reales de AF de la población ${ }^{18}$, lo cual podría 
ocultar la verdadera asociación entre AF y prevalencia de FRCV. Se sugiere que futuras encuestas o evaluaciones nacionales debieran incorporar la medición objetiva de AF, usando, por ejemplo, acelerómetros de movimiento, que ya han sido utilizados en población chilena ${ }^{18,19}$. Otro aspecto que es necesario considerar tiene relación a la limitación del cuestionario GPAQ para estimar ejercicios de fuerza, flexibilidad o equilibrio, que ya han sido incorporados dentro de las recomendaciones de actividad física para la salud. A pesar que la ENS 2009-10 carece de un diseño de seguimiento longitudinal o de estudio de control aleatorio, que no permite establecer una relación de causa-efecto, las asociaciones observadas en este trabajo han sido también reportadas en estudios longitudinales ${ }^{13,20-22}$.

En conclusión, los resultados de este estudio son relevantes en el ámbito de la salud pública, pudiendo servir como base para la implementación de políticas orientadas a la promoción de estilos de vida saludable en la población chilena. La evidencia entregada muestra que la adopción de AF moderada o intensa presenta importantes beneficios en la reducción de la prevalencia de FRCV, sin desconocer además, que actividades como caminar o andar en bicicleta también se asocian a importantes reducciones en el riesgo cardiovascular; en consecuencia, concientizar a la población respecto a los beneficios de incorporar estas actividades dentro de los estilos de vida de la población, podría ser clave en el control y prevención de los FRCV.

Agradecimientos: Se agradece de manera especial a todos los participantes de la ENS 2009-10, como también así al equipo profesional de la Escuela de Salud Pública de la Facultad de Medicina de la Pontífice Universidad Católica de Chile, quienes desarrollaron y aplicaron la Encuesta Nacional de Salud y al Ministerio de Salud del Gobierno de Chile.

\section{Referencias}

1. Lee IM, Shiroma EJ, Lobelo F, Puska P, Blair SN, Katzmarzyk PT, et al. Effect of physical inactivity on major non-communicable diseases worldwide: an analysis of burden of disease and life expectancy. Lancet 2012; 380 (9838): 219-29.

2. WHO. Global health risks: mortality and burden of disease attributable to selected major risks. WHO, 2009.

3. Morris JN, Heady JA, Raffle PA, Roberts CG, Parks JW. Coronary heart-disease and physical activity of work. Lancet 1953; 265 (6796): 1111-concl.

4. Hallal PC, Andersen LB, Bull FC, Guthold R, Haskell W, Ekelund U. Global physical activity levels: surveillance progress, pitfalls, and prospects. Lancet 2012; 380 (9838): 247-57.

5. MINSAL. Encuesta Nacional de Salud 2009-2010. Chile: Ministerio de Salud, 2010.

6. Alberti KGMM, Eckel RH, Grundy SM, Zimmet PZ, Cleeman JI, Donato KA, et al. Harmonizing the Metabolic Syndrome A Joint Interim Statement of the International Diabetes Federation Task Force on Epidemiology and Prevention; National Heart, Lung, and Blood Institute; American Heart Association; World Heart Federation; International Atherosclerosis Society; and International Association for the Study of Obesity. Circulation 2009; 120 (16): 1640-5.

7. WHO. Global Physical Activity Questionnaire: GPAQ version 2.0. World Health Organization, 2009.

8. Bull FC, Maslin TS, Armstrong T. Global Physical Activity Questionnaire (GPAQ): Nine Country Reliability and Validity Study. J Phys Act Health 2009; 6 (6): 790-804.

9. Hoos T, Espinoza N, Marshall S, Arredondo EM. Validity of the Global Physical Activity Questionnaire (GPAQ) in Adult Latinas. J Phys Act Health 2012; 9 (5): 698-705.

10. Ainsworth BE, Haskell WL, Herrmann SD, Meckes N, Bassett DR Jr, Tudor-Locke C, et al. Compendium of Physical Activities: A Second Update of Codes and MET Values. Med Sci Sports Exerc 2011; 43 (8): 1575-81.

11. WHO. Global recommendations on physical activity for health. World Health Organization, 2010.

12. Manson JE, Greenland P, LaCroix AZ, Stefanick ML, Mouton CP, Oberman A, et al. Walking compared with vigorous exercise for the prevention of cardiovascular events in women. NEJM 2002; 347 (10): 716-25.

13. Shiroma EJ, Lee IM. Physical Activity and Cardiovascular Health Lessons Learned From Epidemiological Studies Across Age, Gender, and Race/Ethnicity. Circulation 2010; 122 (7): 743-52.

14. Saevereid HAS, Schnohr PS, Prescott EP. Speed and duration of walking and other leisure time physical activity and the risk of heart failure: the Copenhagen City Heart study. Eur Heart J 2013; 34: 658-.

15. Nocon M, Hiemann T, Mueller-Riemenschneider F, Thalau F, Roll S, Willich SN. Association of physical activity with all-cause and cardiovascular mortality: a systematic review and meta-analysis. Eur J Cardiovasc Prev Rehabil 2008; 15 (3): 239-46.

16. Tudor-Locke C, Craig CL, Brown WJ, Clemes SA, De 
Cocker K, Giles-Corti B, et al. How Many Steps/day are Enough? for Adults. Int J Behav Nutr Phys Act 2011; 8: 79.

17. Cristi-Montero C, Rodríguez FR. The paradox of being physically active but sedentary or sedentary but physically active. Rev Med Chile 2014; 142 (1): 72-8.

18. Celis-Morales CA, Pérez-Bravo F, Ibáñez L, Salas C, Bailey ME, Gill JM. Objective vs. self-reported physical activity and sedentary time: effects of measurement method on relationships with risk biomarkers. PloS one 2012; 7 (5): e36345.

19. Celis-Morales CA, Pérez-Bravo F, Ibáñez L, Sanzana R, Hormazábal E, Ulloa N, et al. Insulin Resistance in Chileans of European and Indigenous Descent: Evidence for an Ethnicity x Environment Interaction. Plos One 2011;
$6(9)$.

20. Ahmed HM, Blaha MJ, Nasir K, Rivera JJ, Blumenthal RS. Effects of physical activity on cardiovascular disease. Am J Cardiol 2012; 109 (2): 288-95.

21. Balducci S, Zanuso S, Cardelli P, Salvi L, Bazuro A, Pugliese L, et al. Effect of High-versus Low-Intensity Supervised Aerobic and Resistance Training on Modifiable Cardiovascular Risk Factors in Type 2 Diabetes; The Italian Diabetes and Exercise Study (IDES). PloS one 2012; 7 (11).

22. Chomistek AK, Manson JE, Stefanick ML, Lu B, Sands-Lincoln M, Going SB, et al. Relationship of Sedentary Behavior and Physical Activity to Incident Cardiovascular Disease. J Am Coll Cardiol 2013; 61 (23): 2346-54. 